\title{
Dual wavelength single longitudinal mode Ytterbium-doped fiber laser using a dual-tapered Mach-Zehnder interferometer
}

H. Ahmad

M. A. M. Salim

S. R. Azzuhri

M. Z. Zulkifli

S. W. Harun
Photonics Research Centre (PRC), University of Malaya

Photonics Research Centre (PRC), University of Malaya

Advanced Photonics Science Institute (APSI), Universiti Teknologi Malaysia

Photonics Research Centre (PRC), University of Malaya

Photonics Research Centre (PRC), University of Malaya

Photonics Research Centre (PRC), University of Malaya

This paper describes a dual wavelength single longitudinal mode (SLM) demonstration for a proposed ytterbium-doped fiber laser. A dualtapered Mach-Zehnder interferometer (MZI) was inserted into the laser ring cavity setup to ensure a stable dual wavelength and SLM operation. The consequent dual wavelength lasing operation had a wavelength spacing of $0.94 \mathrm{~nm}$ and a side mode suppression ratio (SMSR) of $50 \mathrm{~dB}$, with the linewidth of this setup measured as $294.15 \mathrm{kHz}$. A stability test allowed for a measurement of max power fluctuation as less than $0.8 \mathrm{~dB}$ for each wavelength and which was indicative of a stable dual wavelength operation.

[DOI: http://dx.doi.org/10.2971/jeos.2015.15013]

Keywords: Dual wavelength, fiber laser, single longitudinal mode, mach-zehnder

\section{I NTRODUCTION}

Development of dual wavelength fiber lasers (DWFLs) has gained much attention in recent years due to the needs of applications such as photonic generation of microwave carriers [1], microwave photonic filters [2] and high-bit-rate soliton pulses [3], that can only be satisfied by DWFLs. Most of the research in dual wavelength lasers within the last decade has utilized a gain medium of erbium-doped fiber (EDF), which results in emitted wavelengths of $1550 \mathrm{~nm}$. Various proposals for dual wavelength laser generation exploiting EDF, including polarization hole burning [4], the use of fiber Bragg gratings (FBG) [5] and introduction of a filter [6, 7], are readily available in literature. However, there are far less reports on dual wavelength laser generation in the $1 \mu \mathrm{m}$ region using ytterbium-doped fiber (YDF) as the gain medium [8]-[10], which is the interest of this paper.

Most of the proposed setups for DWFL with a YDF gain medium report the use of a FBG as a wavelength selection filter in order to obtain the dual wavelength output [11, 12]. There is also a report describing an arrayed waveguide grating used together with an optical channel selector to produce a stable dual wavelength output [13]. However, dual and multiwavelength output for YDF reported so far has operated under multimode oscillation and mode hopping $[9,10]$ and as such has a severe disadvantage within applications such as optical coherence tomography (OCT) [14] that require a superior coherence and optical properties possessing extremely low noise. Such requirements are only achievable via single longitudinal mode (SLM) operation. SLM in YDF lasers may also be used for achieving second harmonic generation (SHG) [15] if lasing power is sufficiently large, and for optical wireless transmission with $830 \mathrm{~nm}$ laser sources [16]. Several methods have been reported so far to achieve SLM operation using YDF as a gain medium; one report [16] suggested the use of passive multiple-ring cavity configuration to obtain SLM operation in the $1 \mu \mathrm{m}$ range, while another report demonstrated SLM operation using a semiconductor optical amplifier (SOA) and unpumped YDF that acted as a narrowband filter. The only report [17] currently available addressing simultaneous multi-wavelength and SLM operation describes a demonstration of these simultaneous operations through the use of a Sagnac loop mirror. A high level of interest exists for dual wavelength SLM operation involving YDF due to potential applications in optical communication and optical sensing in the $1 \mu \mathrm{m}$ region.

In this paper, a simple YDF laser with a dual-tapered MachZehnder interferometer (MZI) fiber incorporated within a ring cavity setup to obtain simultaneous DWFL and SLM operation is proposed and demonstrated. Dual wavelength and SLM operation was achieved with utilization of the dualtapered MZI fiber and careful adjustments to the light polarization state. The dual wavelength output possessed a $0.94 \mathrm{~nm}$ spacing and a side-mode suppression ratio (SMSR) of $50 \mathrm{~dB}$, while the achieved SLM operation had a linewidth of each wavelength determined as $294.15 \mathrm{kHz}$ via a self-heterodyne calculation method. 


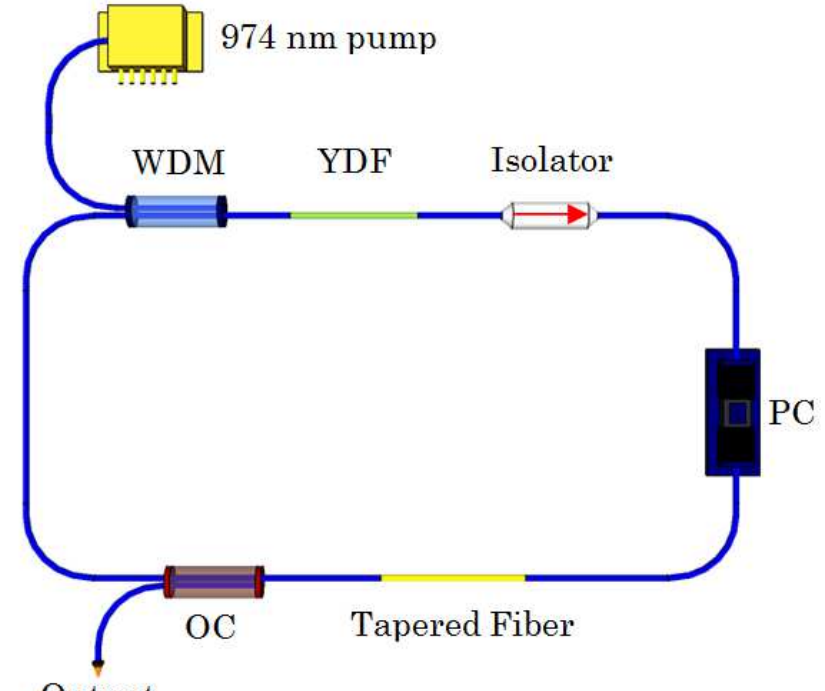

(a)

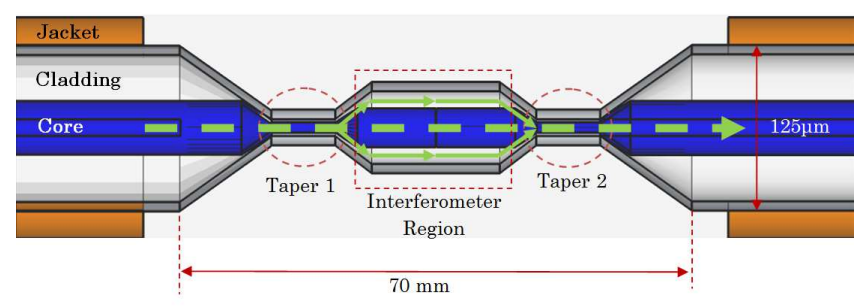

(b)

FIG. 1 (a) experimental setup of dual wavelength YDF laser, and (b) schematic diagram of non-adiabatic tapered fiber.

\section{EXPERIMENTAL SETUP}

The experimental setup of the proposed dual wavelength generation using a dual-tapered MZI fiber is shown in Figure 1 . The fiber ring consisted of a $974 \mathrm{~nm}$ central wavelength pump laser diode with $600 \mathrm{~mW}$ output power (Oclaro model LC96A74P-20R) connected to a 980/1060 nm wavelength division multiplexing (WDM) coupler. One port of the WDM was fusion spliced to a gain medium comprising $70 \mathrm{~cm}$ YDF (DF1100 Fibercore) that had peak absorption of $1300 \mathrm{~dB} / \mathrm{m}$ at $977 \mathrm{~nm}$, and another port was connected to a 90/10 optical coupler. Output of the YDF amplifier was then fusionspliced to a polarization insensitive isolator operating at the $1 \mu \mathrm{m}$ range. This isolator was incorporated into the laser cavity to ensure unidirectional laser ring operation, which was in the clockwise direction in the context of the Figure 1 layout. The output of the isolator was subsequently connected to a polarization controller (PC) that controlled the cavity state of polarization, and the PC output was attached to the dualtapered MZI fiber, which was in turn connected to a 90/10 fused bi-conical optical coupler. This dual-tapered MZI fiber acted as a multi-modal interference medium in order to form a narrow band filter. The $10 \%$ end of the coupler was then connected to the input of an optical spectrum analyser (OSA) of type Yokogawa AQ6373 with a spectral resolution of $0.02 \mathrm{~nm}$.

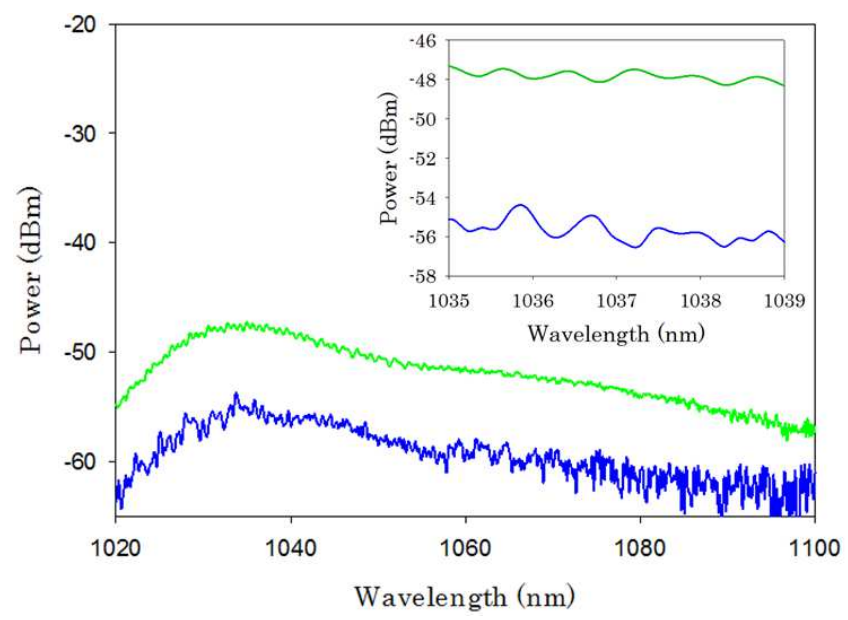

(a)

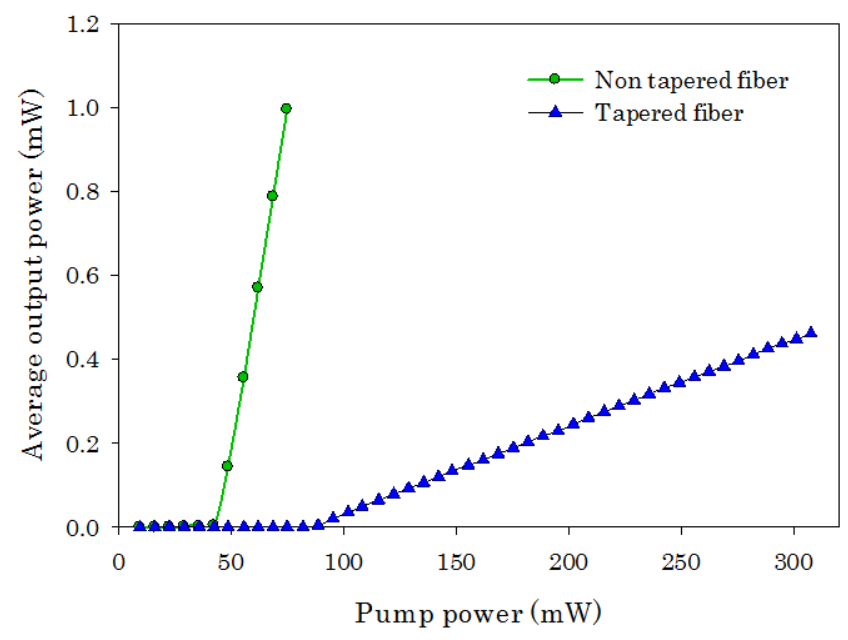

(b)

FIC. 2 (a) ASE spectrum, and (b) slope efficiency of lasing threshold, for tapered fiber (blue) and non tapered fiber (green).

A cross-section overview of the dual-tapered MZI fiber is shown in Figure 1(b) in which the light is considered as propagating from left to right. The fiber jacket was removed from the region intended for tapering, and the heat and pull method [7] was applied in order to fabricate tapered fiber. Two areas, each being approximately $1 \mathrm{~cm}$ in length, were tapered to the core level and are depicted in Figure 1(b) as taper 1 and taper 2. These two sections acted as multimode fiber due to the large difference between the core and air refractive indices. Details regarding the optical properties of this dual-tapered MZI fiber can be obtained in [7], while transmission spectrum of the MZI filter with a non-polarized amplified spontaneous emission (ASE) source is depicted in Figure 2(a). The significant interference pattern, as observed clearly in the blue line of Figure 2, was responsible for the narrow lasing when the ring laser was closed. Lasing usually occurred at the interferences peaks, and simple adjustment of the polarization using a PC caused lasing to shift to another peak, or activated multipeak lasing if supported by the cavity. The lasing threshold for this setup was $88.6 \mathrm{~mW}$, as shown in Figure 2(b), with a total cavity length of $6 \mathrm{~m}$. 


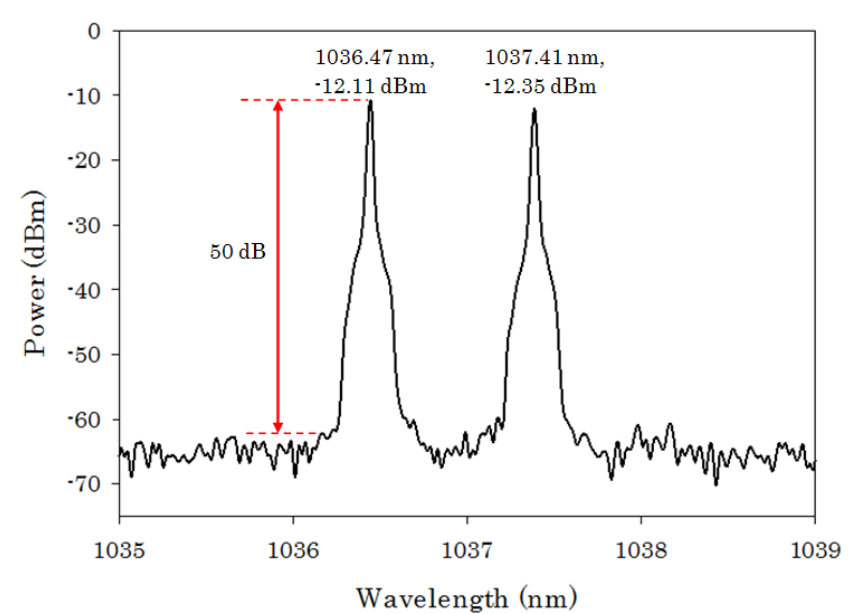

(a)

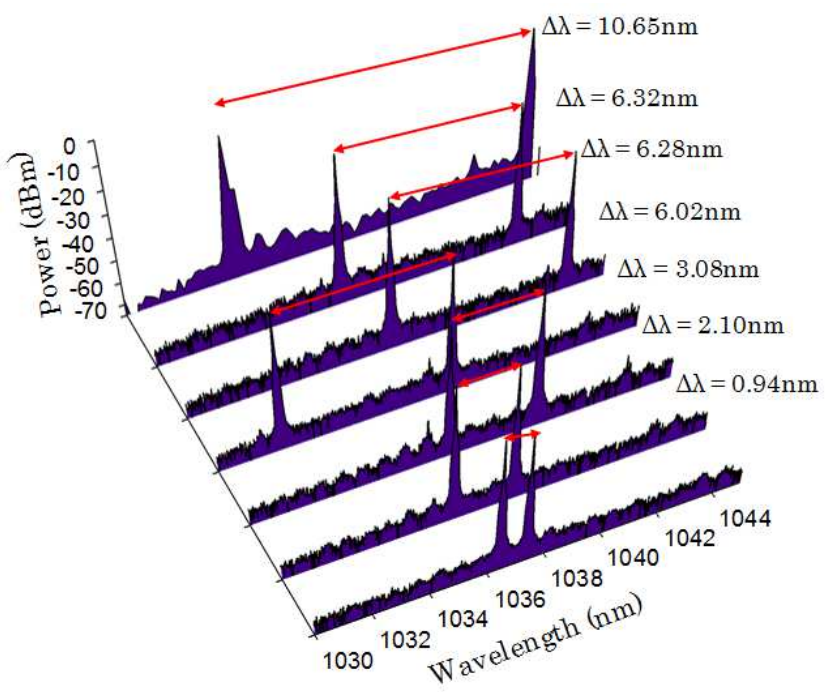

(b)

FIC. 3 (a) dual-wavelength lasing spectrum, and (b) dual-wavelength spectrums with tunable spacing of

(i) $0.94 \mathrm{~nm}$ at $\lambda_{1}=1036.47 \mathrm{~nm}$ and $\lambda_{2}=1037.41 \mathrm{~nm}$

(ii) $2.10 \mathrm{~nm}$ at $\lambda_{1}=1036.03 \mathrm{~nm}$ and $\lambda_{2}=1038.13 \mathrm{~nm}$

(iii) $2.10 \mathrm{~nm}$ at $\lambda_{1}=1036.03 \mathrm{~nm}$ and $\lambda_{2}=1038.13 \mathrm{~nm}$

(iv) $3.08 \mathrm{~nm}$ at $\lambda_{1}=1037.05 \mathrm{~nm}$ and $\lambda_{2}=1040.13 \mathrm{~nm}$

(v) $6.02 \mathrm{~nm}$ at $\lambda_{1}=1032.27 \mathrm{~nm}$ and $\lambda_{2}=1038.29 \mathrm{~nm}$

(vi) $6.28 \mathrm{~nm}$ at $\lambda_{1}=1037.31 \mathrm{~nm}$ and $\lambda_{2}=1043.59 \mathrm{~nm}$

(vii) $6.32 \mathrm{~nm}$ at $\lambda_{1}=1036.65 \mathrm{~nm}$ and $\lambda_{2}=1042.97 \mathrm{~nm}$

(vii) $10.65 \mathrm{~nm}$ at $\lambda_{1}=1033.9 \mathrm{~nm}$ and $\lambda_{2}=1044.55 \mathrm{~nm}$

\section{RESULTS AND DISCUSSION}

Figure 3(a) shows the optical spectrum of the dual wavelength output obtained from the dual-tapered MZI YDF laser ring cavity setup. Spectral shifting of light was accomplished by adjusting the PC so as to change polarization states when light entered the ring cavity. Tuning of the PC caused a rotation of the polarization states and allowed for continuous adjustment of the birefringence within the ring cavity that acted to balance the gain and loss of the lasing wavelengths. The dual wavelength output obtained from the experiment was measured as $1036.47 \mathrm{~nm}$ and $1037.41 \mathrm{~nm}$ with a wavelength spacing of 0.94 $\mathrm{nm}$, while observed SMSR was approximately $50 \mathrm{~dB}$.

Additionally, the dependence of the interference peaks on the

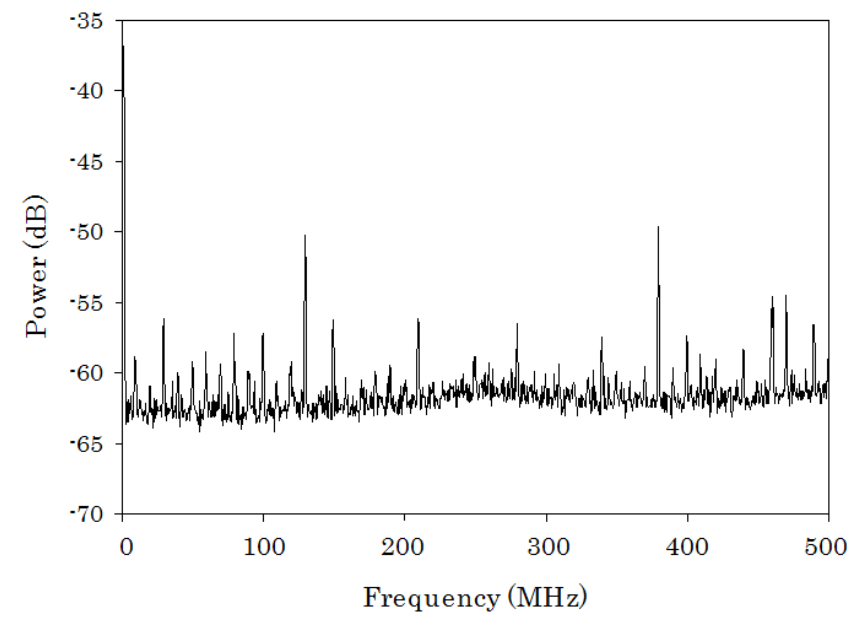

(a)

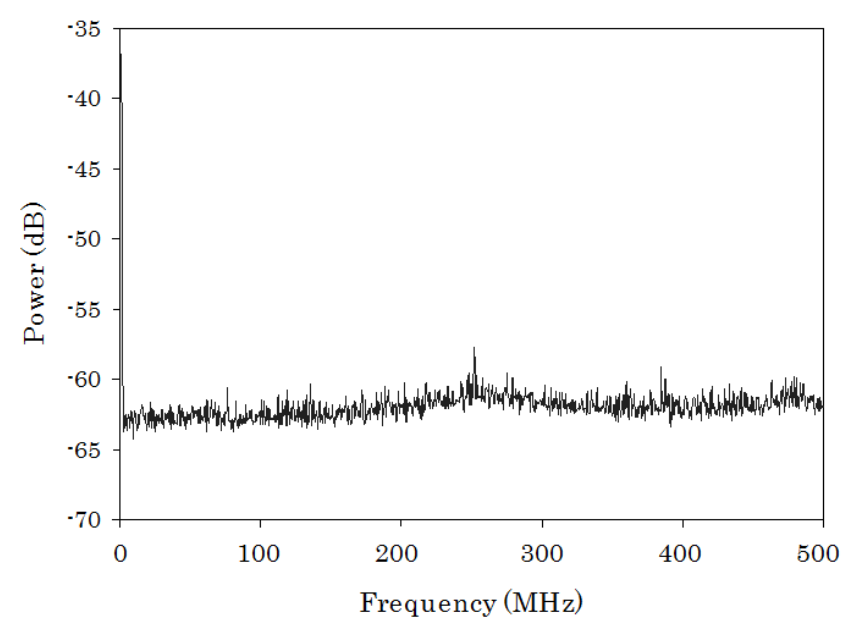

(b)

FIG. 4 RF spectrum of the cavity with (a) dual-tapered fiber disconnected, and (b) dual-tapered fiber connected.

cavity state of polarization (SOP) meant a slight adjustment to the PC caused the wavelength of lasing to switch over to another wavelength as shown in Figure 3(b). The seven acquired sets of dual-wavelength lasing spectrum have a spacing tuning range between $0.94 \mathrm{~nm}$ and $10.65 \mathrm{~nm}$.

Verification of the SLM operation in the proposed setup was undertaken by connecting the $10 \%$ output from the coupler to a RF analyzer (Anritsu MS2683A with a detection range from $9 \mathrm{KHz}$ to $7.8 \mathrm{GHz}$ ) via a Thorlabs D400FC fiber optic photodetector in order to analyse the beating signal of the spectrum. An initial observation of the beating mode, wherein the dualtapered fiber was disconnected from the cavity setup, across a scan range from 0 to $500 \mathrm{MHz}$ is depicted in Figure 4(a). It can be seen that the spectrum is very noisy and unstable due to mode-hopping within the cavity. Connecting the dualtapered fiber to the cavity setup caused the beating noise to disappear, with no mode-hopping observed in the resulting spectrum shown in Figure 4(b).

Measurement of the SLM full width at half maximum (FWHM) linewidth involved application of the heterodyne 


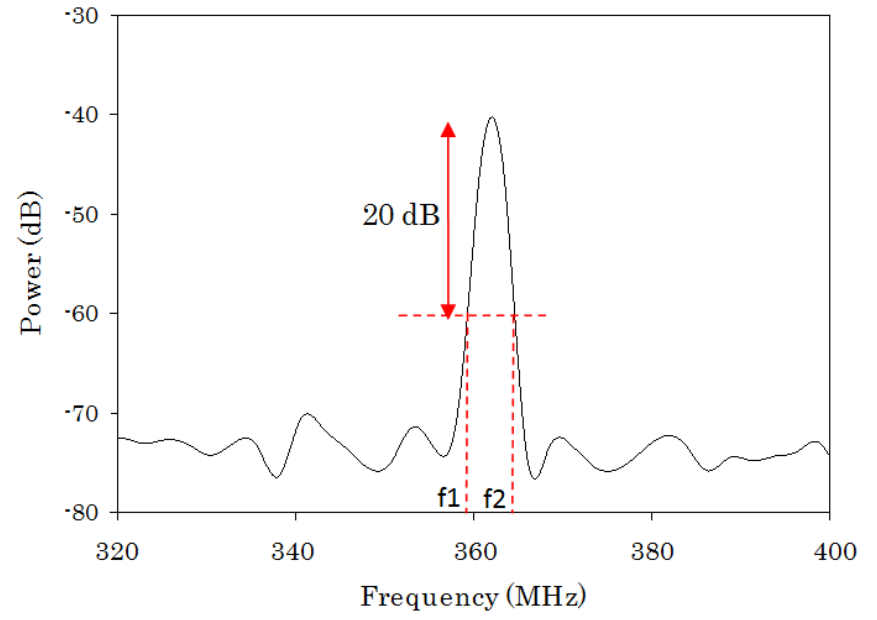

FIG. 5 measured FWHM linewidth spectrum using a heterodyne linewidth method

technique linewidth relations method described in [18] and given by the following equation:

$$
\sqrt{99} \Delta v=\Delta f_{20 \mathrm{~dB}}
$$

where $\Delta v$ is the FWHM linewidth and $\Delta f_{20 \mathrm{~dB}}$ is the frequency difference for a measured full-width point at $20 \mathrm{~dB}$. The measured FWHM linewidth resulting from the heterodyne method is shown in Figure 5. Applying the equation in 1 $f_{2}=3.63 \times 10^{8} \mathrm{~Hz}$ and $f_{1}=3.59 \times 10^{8} \mathrm{~Hz}$ resulted in a $\Delta v$ linewidth value of $294.15 \mathrm{kHz}$, thus providing a verification of SLM operation in the experiment.

All the obtained dual wavelength sets were stability tested over time due to power stability being one of the most important characteristics of DWFL. Figure 6 shows a dual wavelength stability scan for a period of 30 minutes incorporating intervals of 3 minutes for each scan over a total of 10 iterations. Figure 6(a) displays a very stable lasing wavelength over the test period and Figure 6(b) illustrates power fluctuation during the scan, in which the maximum fluctuation is observed as less than $0.8 \mathrm{dBm}$. Such results proved that the wavelength and average power were stable over time and experienced only a very small power fluctuation at room temperature. It should be noted that a test measurement of the dual wavelength was performed simultaneously with these stability tests, and as such these results confirmed the stability and reliability of the proposed setup.

In comparison to the similar approach for SLM operation with YDF reported in [17], the proposal demonstrated in this paper achieved a comparable SMSR and a higher linewidth in the $\mathrm{kHz}$ range while utilizing a simpler setup and less equipment for SLM output. In regards to dual wavelength laser operations, the approach discussed in this paper has a similar SMSR and power stability to the work presented in [11, 12] that used FBG as a wavelength selective filter. Fabrication of FBG requires expensive equipment such as UV laser and phase mask [19] when compared to the low cost and reliable dual-tapered MZI component fabricated in-house. Therefore, the proposed dual-tapered MZI fiber is shown to be an ex-

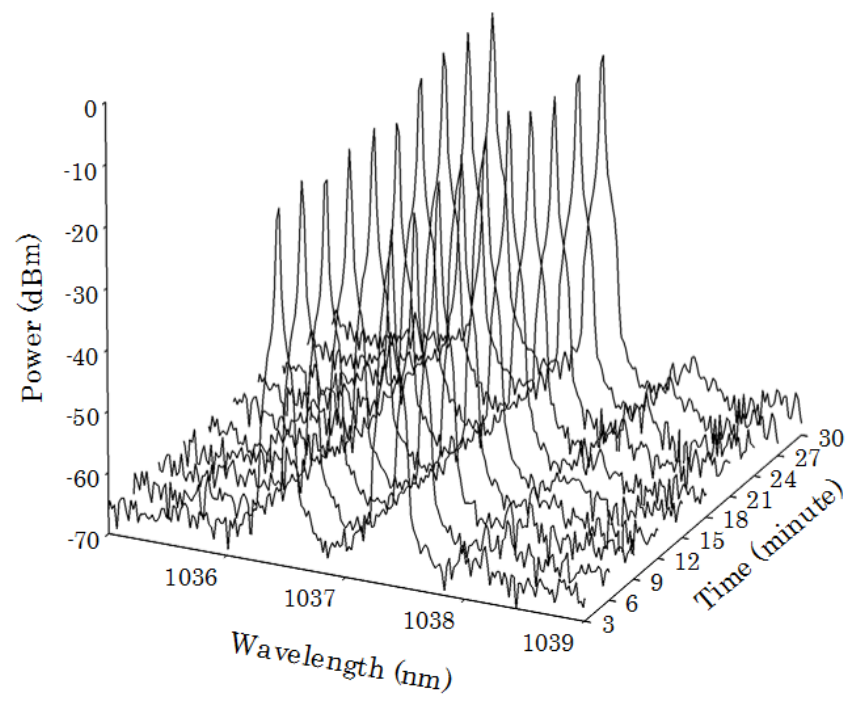

(a)

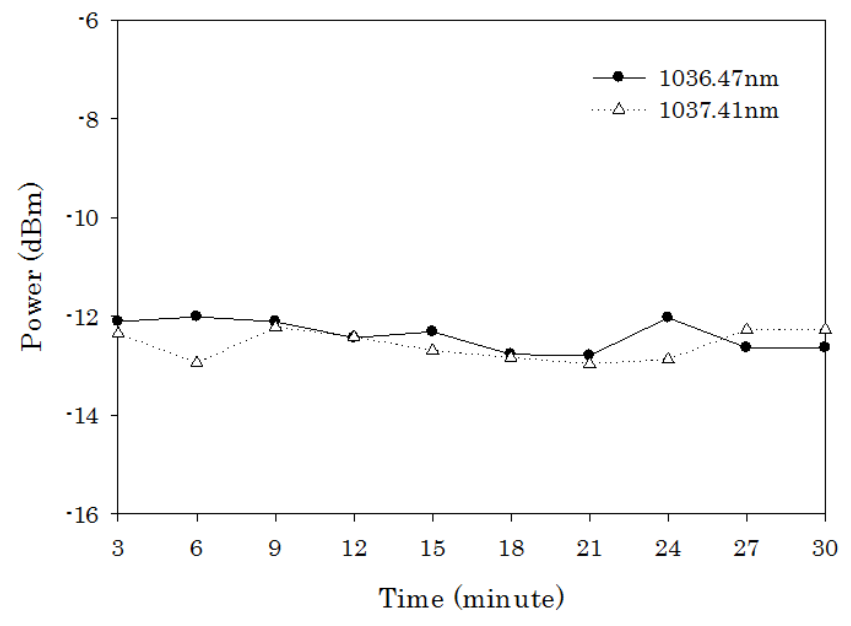

(b)

FIC. 6 (a) dual wavelength output scan, and (b) peak power stability test for spectral spacing of $0.94 \mathrm{~nm}$ with $\lambda_{1}=1036.47 \mathrm{~nm}$ and $\lambda_{2}=1037.41 \mathrm{~nm}$.

cellent choice as a wavelength filter, and has suitability for applications such as OCT, micromachining and optical wireless [20].

\section{CONCLUSIONS}

This paper describes in detail a successful demonstration of a dual wavelength generation and SLM operation utilizing YDF and a dual-tapered fiber MZI filter. Fine-tuning the PC in the proposed setup resulted in generation of dual wavelength lasing with measured wavelength spacing of $0.94 \mathrm{~nm}$ and a SMSR of approximately 50dB. The SLM linewidth resulting from the experiment was calculated via the heterodyne technique as being $294.15 \mathrm{kHz}$, while a stable dual wavelength output with maximum power fluctuation of $0.8 \mathrm{dBm}$ over a period of 30 minutes was achieved. These results mean that the proposed setup relative to similar reported approaches has key advantages of simpler setup, lower cost deployment, and highly stable operation under room temperature for the $1 \mu \mathrm{m}$ wavelength region. The authors of this paper anticipate such findings presented here will spur further developments and applications in this area. 


\section{ACKN OWLEDGMENTS}

We would like to thank university of Malaya for the research funding, under grant number RU 002/2013, RP008A-13AET and RG143-12AET.

\section{References}

[1] L. Xia , P. Shum, and T. H. Cheng, "Photonic generation of microwave signals using a dual-transmission-band FBG filter with controllable wavelength spacing," Appl. Phys. B-Lasers 0. 86, 61-64 (2007).

[2] D. Liu, N. Q. Ngo, G. Ning, P. Shum, and S. C. Tjin, "Tunable microwave photonic notch filter using a dual-wavelength fiber laser with phase modulation," Opt. Commun. 266, 240-248 (2006).

[3] M. Tadakuma, 0. Aso, and S. Namiki, $104 \mathrm{CHz} 328$ fs soliton pulse train generation through a comb-like dispersion profiled fiber using short high nonlinearity dispersion fibers (Optical Fiber Communication Conference, Maryland, 2000).

[4] J. R. Qian, J. Su, and L. Hong, "BA widely tunable dual-wavelength erbium-doped fiber ring laser operating in single longitudinal mode," Opt. Commun. 281, 44321-4434 (2008).

[5] F. Zhang, P. L. Chu, R. Lai, and G. R. Chen, "Dual-wavelength chaos generation and synchronization in erbium-doped fiber laser," IEEE Photonic. Tech. L. 17, 549-551 (2005).

[6] X. Feng, Y. Liu, S. Yuan, G. Kai, W. Zhang, and X. Dong, "L-Band switchable dual- wavelength erbium-doped fiber laser based on a multimode fiber Bragg grating," Opt. Express 12, 3834-3839 (2004).

[7] H. Ahmad, M. Dernaika, 0. M. Kharraz, M. Alimadad, M. F. Ibrahim, K. S. Lim, and S. W. Harun, "A tuneable, power efficient and narrow single longitudinal mode fibre ring laser using an inline dual-taper fibre Mach-Zehnder filter," Laser Phys. 24, 085111 (2014).

[8] P. Zhou, X. Wang, H. Xiao, Y. Ma, and J. Chen, “Review on recent progress on $\mathrm{Yb}$-doped fiber laser in a variety of oscillation spectral ranges," Laser Phys. 22, 823-831 (2012).

[9] M. R. A. Moghaddam, S. W. Harun, M. C. Paul, M. Pal, A. Dhar, R. Sen, S. Das, et al., "Multiwavelength ytterbium-doped fiber ring laser," Microw. Opt. Techn. Let. 51, 25111-2512 (2009).

[10] R. Chi, K. Lu, and S. Chen, "Multiwavelength Yb-doped fiber ring laser," Microw. Opt. Techn. Let. 36, 1701-172 (2003).
[11] H. Wang, Y. G. Li, X. D. Chen, B. Huang, F. Y. Lu, and K. C. Lu, "Highly efficient dual-wavelength ytterbium-doped fiber linear cavity laser based on cascaded fiber Bragg gratings," Laser Phys. 19, 1257-1262 (2009).

[12] X. Feng, Y. Liu, S. Fu, S. Yuan, and X. Dong, "Switchable dualwavelength ytterbium-doped fiber laser based on a few-mode fiber grating," IEEE Photonic. Tech. L. 16, 762-764 (2004).

[13] H. Ahmad, A. A. Latif, S. F. Norizan, M. Z. Zulkifli, and S. W. Harun, "Flat and compact switchable dual wavelength output at 1060nm from ytterbium doped fiber laser with an AWG as a wavelength selector," Opt. Laser Technol. 43, 550-554 (2011).

[14] F. Yin, S. Yang, H. Chen, M. Chen, and S. Xie, "60-nm-wide tunable single-longitudinal-mode ytterbium fiber laser with passive multiple-ring cavity," IEEE Photonic. Tech. L. 23, 16581-1660 (2011).

[15] 0. B. Jensen, P. E. Andersen, B. Sumpf, K. H. Hasler, G. Erbert, and P. M. Petersen, "1.5W green light generation by single-pass second harmonic generation of a single-frequency tapered diode laser," Opt. Express 17, 65321-6539 (2009).

[16] S. Rajbhandari, Z. Chassemlooy, and J. Perez, "On the study of the FSO link performance under controlled turbulence and fog atmospheric conditions" in Proceedings of the 2011 11th international conference on telecommunications, 2231-226 (ConTEL, Graz, 2011).

[17] Y. Zhou, P. C. Chui, and K. K. Y. Wong, "Multiwavelength singlelongitudinal-mode ytterbium-doped fiber laser," IEEE Photonic. Tech. L. 25, 385-388 (2013).

[18] D. Derickson, Fiber optic test and measurement (Prentice Hall, New York, 1998).

[19] Y. J. Song, L. Zhan, S. Hu, Q. H. Ye and Y. X. Xia, "Tunable multiwavelength Brillouin-erbium fiber laser with a polarizationmaintaining fiber Sagnac loop filter," IEEE Photonic. Tech. L. 16, 2015-2017 (2004).

[20] Y. L. Yu, S. K. Liaw, W. C. Hsu, M. H. Shih, and N. K. Chen, “Single longitudinal mode Ytterbium doped fiber lasers with large proposed tuning range," Opt. Quant. Electron. 47, 131-137 (2015). 